\title{
Symbiotic microbiome Staphylococcus aureus from human nasal mucus modulates IL-33-mediated type 2 immune responses in allergic nasal mucosa
}

Yung Jin Jeon ${ }^{1}$, Chan Hee Gil ${ }^{2}$, Jina Won ${ }^{2}$, Ara Jo ${ }^{3}$ and Hyun Jik Kim², ${ }^{2}{ }^{*}$

\begin{abstract}
Background: The host-microbial commensalism can shape the innate immune responses in respiratory mucosa and nasal microbiome also modulates front-line immune mechanism in the nasal mucosa. Inhaled allergens encounter the host immune system first in the nasal mucosa, and microbial characteristics of nasal mucus directly impact the mechanisms of initial allergic responses in nasal epithelium. However, the roles of the nasal microbiome in allergic nasal mucosa remain uncertain. We sought to determine the distribution of nasal microbiomes in allergic nasal mucosa and elucidate the interplay between nasal microbiome Staphylococcus species and Th2 cytokines in allergic rhinitis (AR) models.
\end{abstract}

Results: Staphylococcus aureus (AR-SA) and S. epidermidis (AR-SE) were isolated from the nasal mucosa of patients with AR. The influence of nasal microbiome Staphylococcus species on allergic nasal mucosa was also tested with in vitro and in vivo AR models. Pyrosequencing data showed that colonization by S. epidermidis and S. aureus was more dominant in nasal mucus of AR subjects. The mRNA and protein levels of IL-33 and TSLP were significantly higher in AR nasal epithelial (ARNE) cells which were cultured from nasal mucosa of AR subjects, and exposure of ARNE cells to AR-SA reduced IL-33 mRNA and secreted protein levels. Particularly, ovalbumin-driven AR mice inoculated with AR-SA by intranasal delivery exhibited significantly reduced IL-33 in their nasal mucosa. In the context of these results, allergic symptoms and Th2 cytokine levels were significantly downregulated after intranasal inoculation of AR-SA in vivo AR mice.

Conclusion: Colonization by Staphylococcus species was more dominant in allergic nasal mucosa, and nasal commensal S. aureus from subjects with AR mediates anti-allergic effects by modulating IL-33-dependent Th2 inflammation. The results demonstrate the role of host-bacterial commensalism in shaping human allergic inflammation.

Keywords: Staphylococcus aureus, Allergic rhinitis, Interleukin-33, Symbiosis, Nasal microbiome

\footnotetext{
* Correspondence: hyunjerry@snu.ac.kr

${ }^{2}$ Department of Otorhinolaryngology - Head and Neck Surgery, Seoul

National University College of Medicine, 103, Daehak-ro, Jongno-gu, Seoul 03080, Republic of Korea

${ }^{3}$ Department of Otorhinolaryngology, Seoul National University Hospital, Seoul, Republic of Korea

Full list of author information is available at the end of the article
}

(c) The Author(s). 2020 Open Access This article is licensed under a Creative Commons Attribution 4.0 International License, which permits use, sharing, adaptation, distribution and reproduction in any medium or format, as long as you give appropriate credit to the original author(s) and the source, provide a link to the Creative Commons licence, and indicate if changes were made. The images or other third party material in this article are included in the article's Creative Commons. licence, unless indicated otherwise in a credit line to the material. If material is not included in the article's Creative Commons licence and your intended use is not permitted by statutory regulation or exceeds the permitted use, you will need to obtain permission directly from the copyright holder. To view a copy of this licence, visit http://creativecommons.org/licenses/by/4.0/ The Creative Commons Public Domain Dedication waiver (http://creativecommons.org/publicdomain/zero/1.0/) applies to the data made available in this article, unless otherwise stated in a credit line to the data. 


\section{Background}

Allergic rhinitis (AR), an IgE- and T helper (Th)2-mediated inflammatory nasal disease, is caused by sensitized immune responses to inhaled allergens. This allergenspecific immune response is thought to arise from an imbalance in Th1-Th2 immune regulation that results in increased levels of Th2 cytokines [1, 2]. Nasal epithelial cells exposed to external allergens induce Th2 inflammatory responses and Th2 inflammations proceed to the upper airway mucosa. The allergen-mediated inflammatory immune response begins with increased secretion of epithelial cell-derived cytokines, such as thymic stromal lymphopoietin (TSLP) and interleukin (IL)-33 [1]. Epithelial cell-derived cytokines provide critical signals to innate and adaptive cell populations related to Th2 inflammation [2, 3]. Allergic nasal epithelium might be responsible for the vast majority of allergic inflammation to inhaled allergens, and research about regulation of epithelial cell-derived cytokines is needed to develop a more effective approach to treatment of AR [4]. IL-33, which is produced by the airway epithelium and other cell types, is a key cytokine involved in allergic airway diseases and provides an essential axis for rapid immune responses and tissue homeostasis. Since the discovery of IL-33, tremendous progress has been made in understanding its biological functions, and the potential importance of IL-33 as a therapeutic target of AR has been suggested $[5,6]$.

Human mucosal surfaces are in direct contact with the external environment and are susceptible to invasion and colonization by various allergens and pathogens [7-9]. Respiratory mucosa is constantly exposed to inhaled pathogens and allergens that directly impact mucosal immune mechanisms $[10,11]$. Studies on interaction between the mucosal microbiome and the host increasingly consider the contribution of mucosal immune responses and specific microbiome-mediated protection from external pathogens to integrate environmental signals [11]. Inhaled allergens encounter the host immune system first in the nasal mucosa, and microbial characteristics of nasal mucus directly impact the mechanisms of initial allergic responses in nasal epithelium $[9,12,13]$. Insights into the microbiota and dysbiosis of allergic nasal mucosa provide fundamental information regarding susceptibility to allergens and their relation to allergic inflammation, including induction of epithelial cell-derived Th2 cytokines such as IL-33 [14-16]. There is considerable evidence that the microbiota contributes to responses of the mucosal immune system and protects against pathogenic bacterial or viral infection. Recent studies have sought to identify how the commensal microbiota regulates sensitization tolerance to allergens in asthma $[9,17,18]$. However, our knowledge of microbial composition in allergic nasal mucus is limited, and the responses of the nasal microbiome to inhaled allergens have not been comprehensively examined in nasal mucosa.
Based on our previous evidence of microbial distribution in healthy nasal mucus, we assessed the microbial composition in allergic nasal mucus and subsequently investigated whether nasal commensal bacteria in allergic nasal mucus contributes to Th2 inflammatory responses in nasal epithelium. The present study identified Staphylococcus species, including S. epidermidis and $S$. aureus, as the most abundant constituents in allergic nasal mucus. Particularly, S. aureus isolated from the nasal mucus of AR subjects accelerated the reduction of IL-33 in AR nasal epithelium. Furthermore, allergic nasal commensal S. aureus prevented allergic inflammation in vivo in mice by reducing IL-33-related Th2 immune responses in the nasal mucosa. Overall, our study presents evidence that Staphylococcus species colonization is significantly increased in the nasal mucus of AR subjects. In addition, administration of $S$. aureus isolated from human allergic nasal mucus suppressed allergic inflammation in nasal mucosa by reducing IL-33 secretion.

\section{Materials and methods}

Additional methodological details are available in the online supplement.

\section{Participant recruitment}

Information on 17 subjects with AR enrolled in this study and the exclusion criteria are described in the online data supplement. Participation was voluntary, and written informed consent was obtained from all subjects. The Institutional Review Board of Seoul National University College of Medicine (No. 1709-049-883) and Gyeongsang National University Hospital (No. 2019-05004) approved the protocol for this study.

\section{Sample collection}

Mucus and/or nasal mucosa from the middle turbinate of human subjects was collected and assessed for quality as described in the online data supplement (supplementary video file).

\section{The characterization of staphylococcus species in human allergic nasal mucosa}

To isolate bacterial colonies, the mucus was placed in lysogeny broth (LB) plates. After 1 day of incubation, bacterial colonies were obtained from LB plates. The species of each colony was identified using GS-FLX 454 pyrosequencing with $16 \mathrm{~S}$ rRNA gene amplification, as described previously [19]. Staphylococcus epidermidis and $S$. aureus strains from five individual subjects with AR were used in the study.

\section{Cell culture}

Normal human nasal epithelial (NHNE) and allergic rhinitis nasal epithelial (ARNE) cells were each cultured 
from five subjects using an air-liquid interface method. Cells were used 14 days after creation of the air-liquid interface (ALI). Isolated AR-SA and AR-SE were inoculated as described previously [19]. Details are available in the online supplement.

\section{Murine inoculation model}

Experiments with four-week-old female wild type (WT) BALB/c mice (Orient, Gyeonggi, Republic of Korea) were carried out according to guidelines approved by the Institutional Animal Care and Use Committees of Seoul National University Hospital (No. 2016-1470). Microbiome depletion, AR-SA and AR-SE inoculation, nasal lavage (NAL) sample collection, and nasal tissue harvesting are described in the online supplement.

Mice were divided into four groups depending on $S$. aureus inoculation. The negative control group (WT) was sensitized and challenged with phosphate-buffered saline (PBS), and the positive control group (AR-OVA) was sensitized and challenged with ovalbumin (OVA). Both WT mice and AR-OVA mice were inoculated with human nasal microbiome $S$. aureus by intranasal delivery. The schematic figure for allergen sensitization, intranasal challenge, and S. aureus inoculation is shown in Fig. 4a. Mice were euthanized and sacrificed by cervical dislocation and by intramuscular injection of high dose of a mixture of $10 \mathrm{mg} / \mathrm{kg}$ xylazine (Bayer, Puteaux, France) and $5 \mathrm{mg} / \mathrm{kg}$ ketamine (Merial, Lyon, France) according to the reviewed protocol. Death was verified when no heartbeat was detected. There were no mice without euthanasia. When mice were euthanized by injection, a cervical dislocation was also performed to ensure that the mice were dead.

\section{Serum levels of total and OVA-specific lgE}

AR mouse serum samples were stored at $-70^{\circ} \mathrm{C}$ before use for measurements of total IgE and OVA specific IgE, as described previously [20]. Details are available in the online supplement.

\section{Histologic analysis}

Mice heads were fixed in 10\% formalin, decalcified, and embedded in paraffin wax. Nasal tissues were sectioned and stained with hematoxylin and eosin (H\&E) for inflammatory cell counting, Sirius red stain for eosinophil counting, and periodic acid-Schiff (PAS) stain to indicate secretory cells of the nasal epithelium.

\section{Colony count}

Bacterial samples were serially diluted ten-fold with phosphate-buffered saline (PBS). Next, $10 \mu \mathrm{l}$ of each diluted sample was plated on an LB agar plate. The plates were incubated for $24 \mathrm{~h}$ at $37^{\circ} \mathrm{C}$. The numbers of AR-SA and AR-SE colonies growing on the agar surface were counted manually. Bacterial growth was reported based on the colony-forming units (CFUs) for each sample.

\section{Real-time PCR and RNA preparation}

Levels of transcripts encoding human IL-33 and TSLP, mouse IL-4, IL-5, IL-13, and IL-33, or femAs specific for S. aureus (femA-SA) and S. epidermidis (femA-SE) were determined using real-time PCR (RT-PCR), as described in the online supplement.

\section{Protein isolation and Western blot}

IL-33 levels were assessed by western blotting using human IL-33 antibody (AF3625, R\&D Systems, Minneapolis, MN, USA). Details are available in the online supplement.

\section{Quantification of secreted cytokines}

Secreted mouse IL-33 (DY3626) was quantified using the DuoSet ${ }^{\circ}$ ELISA kit from R\&D Systems according to the manufacturer's instructions. To quantify IL-33, nasal lavage (NAL) fluid of a murine model was collected. The working range of the assay was $15.6-1000 \mathrm{pg} / \mathrm{mL}$.

\section{Statistical analyses}

At least three independent experiments were performed with cultured cells from each donor, and data are expressed as mean \pm standard deviation (SD) of three independent experiments. Groups were compared by the Mann-Whitney $U$ test, and differences among treatment groups were evaluated by analysis of variance (ANOVA) with a post hoc test. A $P$-value of $<0.05$ was considered statistically significant. All statistical analyses were performed with GraphPad Prism software (version 5; GraphPad Software, Inc., La Jolla, CA, USA).

\section{Results}

Characterization of bacterial communities in allergic nasal mucus

The microbial compositions of middle turbinate mucus in human subjects with $\mathrm{AR}(N=17)$ were evaluated by analyzing cultured bacterial colonies and $16 \mathrm{~S}$ rRNA gene sequencing, as previously described [6]. Based on at least $97 \%$ sequence identity, 112 bacterial species were discovered in middle turbinate mucus of subjects with AR. Staphylococcus epidermidis, S. aureus, Enterobacter aerogenes, Corynebacterium accolens, Micrococcus luteus, Corynebacterium pseudodiphtheriticum, and Klebsiella pneumoniae were among the most commonly detected species. S. epidermidis demonstrated the highest abundance, accounting for $58.04 \%$ of mapped sequences, and $S$. aureus exhibited the largest increase in presence of microbial distribution in allergic nasal mucus (14.29\% in AR vs $5.61 \%$ in healthy nasal mucus [6]) (Fig. 1a). The proportion of $S$. epidermidis and $S$ aureus in allergic nasal mucus varied widely among subjects with AR to a maximum of $79.1 \%$. The lowest abundance of 
A

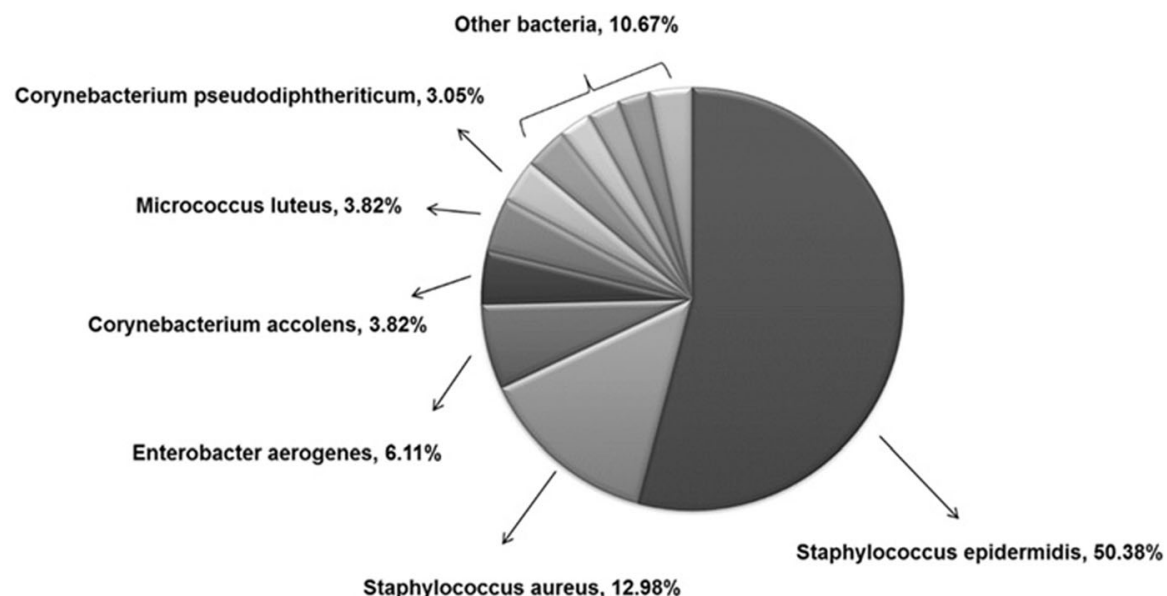

Staphylococcus aureus, $12.98 \%$
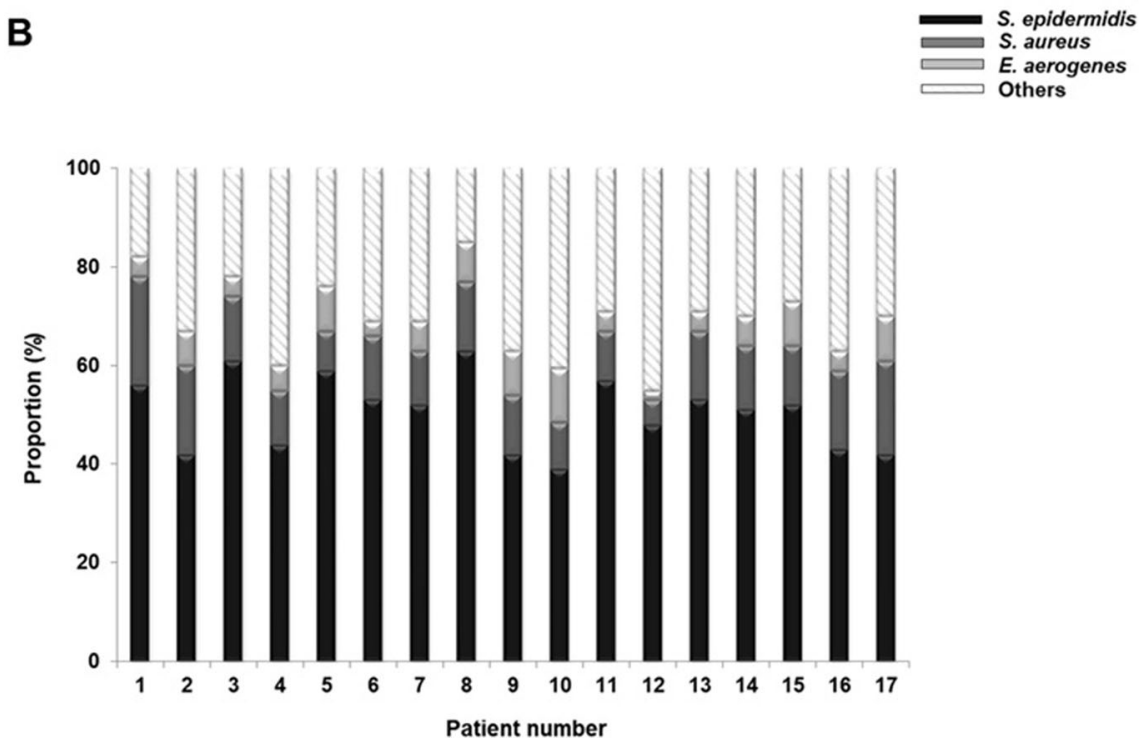

Fig. 1 Distribution of bacterial colonies in nasal mucus of subjects with allergic rhinitis (AR). a Bacterial species cultured from mucus samples obtained from middle turbinates of subjects with allergic rhinitis $(A R)(N=17)$ were identified via $16 S$ rRNA gene sequencing. Distribution of the 112 identified bacterial species is presented in the graph. $\mathbf{b}$ The relative abundance of nasal commensal, such as S. epidermidis, S. aureus, E. aerogenes and others, in each AR subjects. The bar graph presents the relative abundance of nasal commensal organisms of 17 allergic rhinitis subjects at the species level

Staphylococcus species in the nasal mucus of subjects with AR was $48.9 \%$ (Fig. 1b). We then questioned the role of the nasal commensal organisms $S$. epidermidis (AR-SE) and $S$. aureus (AR-SA) in allergic nasal mucus. Both AR-SE and AR-SA strains were isolated from four AR subjects to assess the mechanistic link between dominant allergic nasal commensals and pathogenesis of AR in nasal mucosa.

\section{Inoculation of nasal commensal S. aureus suppressed IL-} 33 secretion in ARNE cells

The kinetics of expression of epithelial cell-derived Th2 cytokines genes, protein production, and secreted protein levels were studied in ARNE cells after inoculation with dominant nasal microbiome AR-SE and AR-SA in human allergic nasal mucosa. Cultured ARNE cells from five subjects with AR were inoculated with AR-SA and AR-SE at an MOI of 0.25 , and cell lysate and supernatant were harvested at $0,2,8,24$, and $48 \mathrm{~h}$ post of inoculation (hpi). The mRNA levels of femA-SA and femA-SE genes were then measured using RT-PCR. The results showed that femA-SA and femA-SE mRNA levels increased significantly from $8 \mathrm{~h}$ after inoculation, with the highest expression observed at 48 hpi (Fig. 2a, b). In addition, AR-SA colony counts from the supernatant of ARNE were significantly increased at $8 \mathrm{hpi}\left(1.05 \times 10^{8} \pm 7.07 \times 10^{6} \mathrm{CFU} / \mathrm{mL}\right)$ and were the highest at $48 \mathrm{hpi}\left(1.28 \times 10^{9} \pm 3.20 \times 10^{8}\right.$ 
A

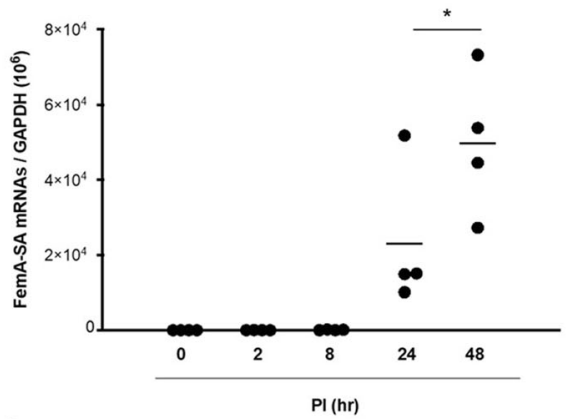

C

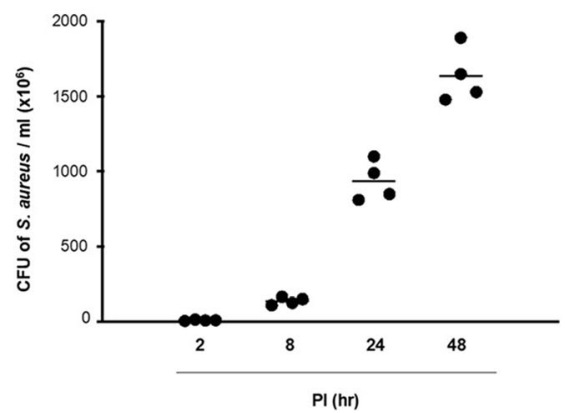

B



D

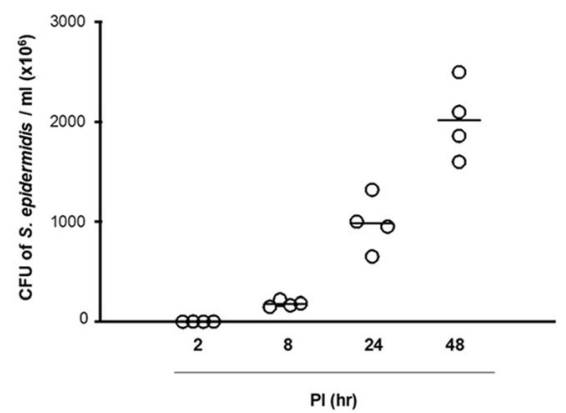

Fig. 2 Susceptibility of nasal commensal Staphylococcus species to allergic rhinitis nasal epithelial (ARNE) cells. ARNE cells from four AR subjects were inoculated with nasal commensal, S. aureus and S. epidermidis at a multiplicity of infection (MOI) of 0.25 . The mRNA levels of femA genes specific for S. aureus (a) and S. epidermidis (b), normalized to cellular GAPDH transcript levels, were monitored by real-time PCR for $48 \mathrm{~h}$ post-infection. Colony counts of supernatants in cultured ARNE cells inoculated with nasal commensal S. aureus (c) and S. epidermidis (d) from allergic rhinitis subjects were performed. Results are presented as mean \pm standard deviation (SD) from five independent experiments. ${ }^{*} p<0.05$ compared with control ARNE cells

CFU/mL) (Fig. 2c). AR-SE colony counts from supernatant of ARNE cells were also significantly increased at 8 hpi $\left(1.35 \times 10^{8} \pm 2.12 \times 10^{7} \mathrm{CFU} / \mathrm{mL}\right)$ and were the highest at $48 \mathrm{hpi}\left(1.85 \times 10^{9} \pm 3.53 \times 10^{8} \mathrm{CFU} / \mathrm{mL}\right)($ Fig. $2 \mathrm{~d})$. Then, mRNA levels of IL-33 and TSLP were measured to analyze the effects of nasal commensal Staphylococcus species inoculation on the expression of epithelial cellderived Th2 cytokines in ARNE cells. Although IL-33 mRNA levels were also slightly attenuated in AR-SE inoculated ARNE cells at $48 \mathrm{hpi}$, IL-33 mRNA levels of ARNE cells decreased more significantly after inoculation of ARSA from $8 \mathrm{hpi}$, and the lowest level was observed in ARSA-inoculated ARNE cells at 48 hpi (Fig. 3a, b). TSLP mRNA levels did not significantly change in ARNE cells inoculated with AR-SA or AR-SE (Fig. 3c, d).

We then tested whether $S$. aureus targets IL-33 gene and protein expression in ARNE cells and compared expression levels in ARNE cells with those of AR-SAinoculated NHNE cells. The IL-33 mRNA levels of ARNE cells were significantly higher than those of NHNE cells regardless of AR-SA inoculation (mean value of ARNE $1.21 \times 10^{8}$ vs mean value of NHNE $\left.3.82 \times 10^{6}\right)$. Reduced $I L-33$ gene expression was only observed in AR-SA inoculated ARNE cells until $48 \mathrm{~h}$ after inoculation (mean value: $3.21 \times 10^{3}$, Fig. 3e). Western blot analysis similarly revealed that full-length IL-33 protein levels gradually decreased until $48 \mathrm{~h}$ in AR-SA-inoculated ARNE cells, and AR-SA did not show any IL-33 reduction in NHNE cells (Fig. 3f). The secreted protein levels of IL-33 in the supernatant of ARNE cells was measured using ELISA and compared with those of NHNE cells after AR-SA inoculation. The secreted protein concentration of IL-33 was significantly higher in the supernatant of ARNE cells (mean value of ARNE: $3693.2 \mathrm{pg} / \mathrm{ml}$ vs mean value of NHNE: $231.4 \mathrm{pg}$ / $\mathrm{ml}$ ) and secreted protein concentration of IL-33 was reduced in the supernatant of AR-SA-inoculated ARNE cells until $48 \mathrm{~h}$ after inoculation (mean value: $103.5 \mathrm{pg}$ / $\mathrm{ml}$, Fig. 3g). Based on these findings, nasal microbiome $S$. aureus isolated from the nasal mucus of subjects with AR reduced epithelial cell-derived Th2 cytokines, IL-33 gene expression, and protein production in allergic nasal epithelium.

Pretreatment with human nasal microbiome $S$. aureus suppresses allergic inflammation in vivo

Considering the in vitro effect of nasal commensal $S$. aureus on IL-33 expression in ARNE cells, we next 

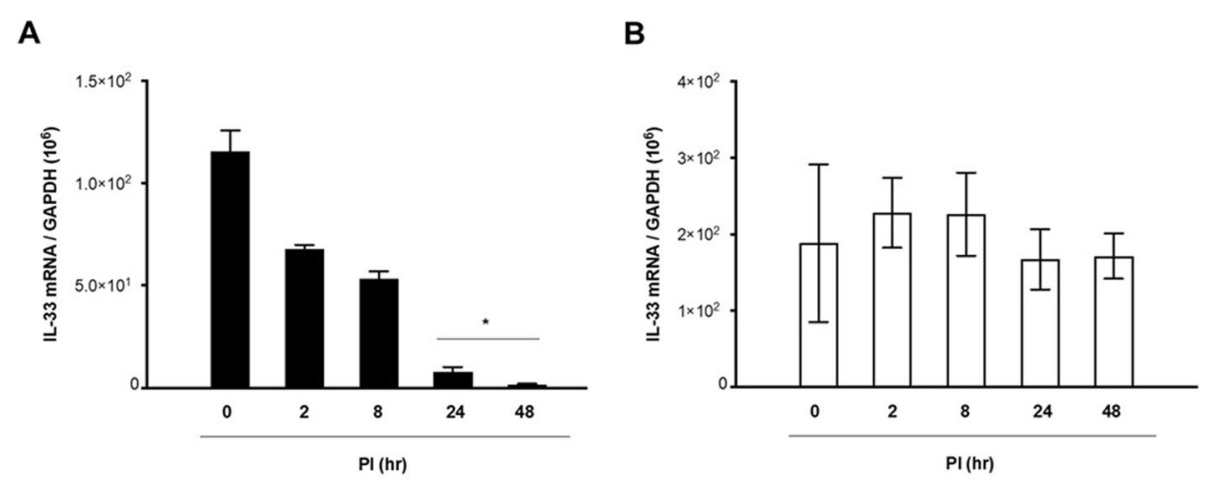

C

D
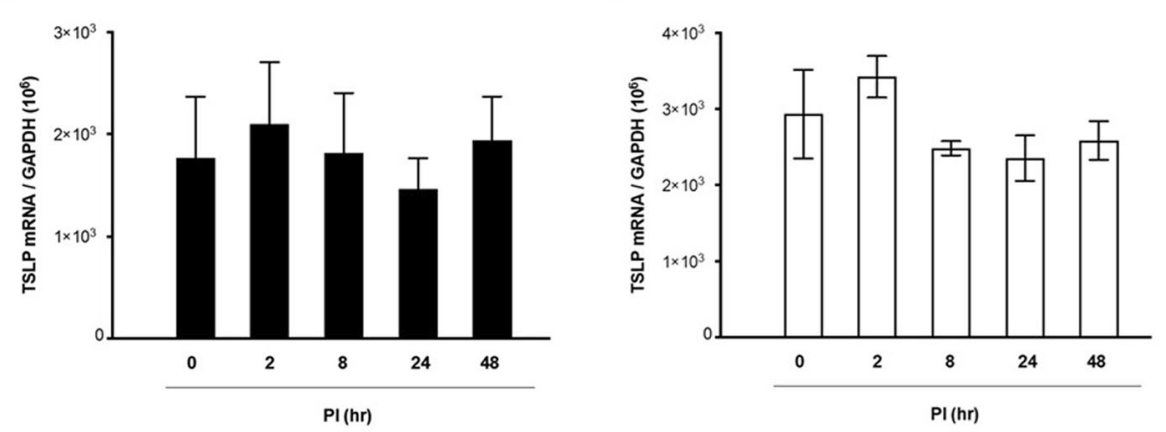

E

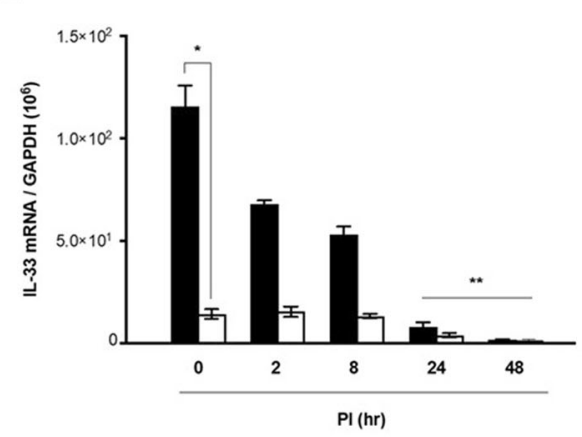

F

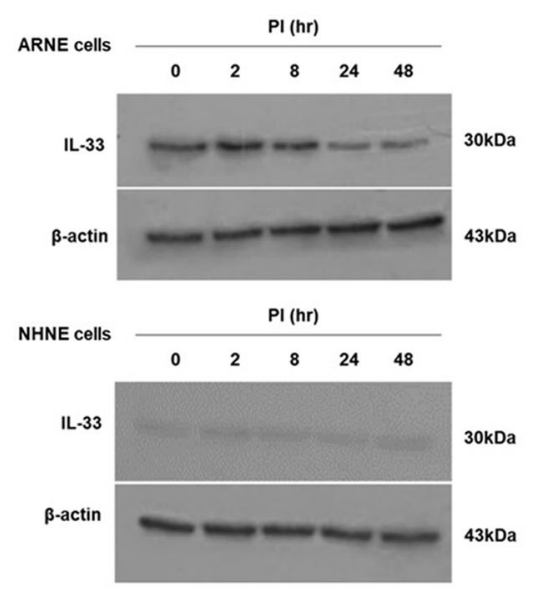

G

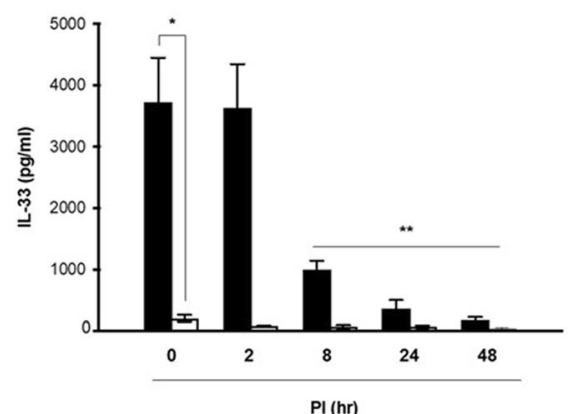

Fig. 3 (See legend on next page.) 


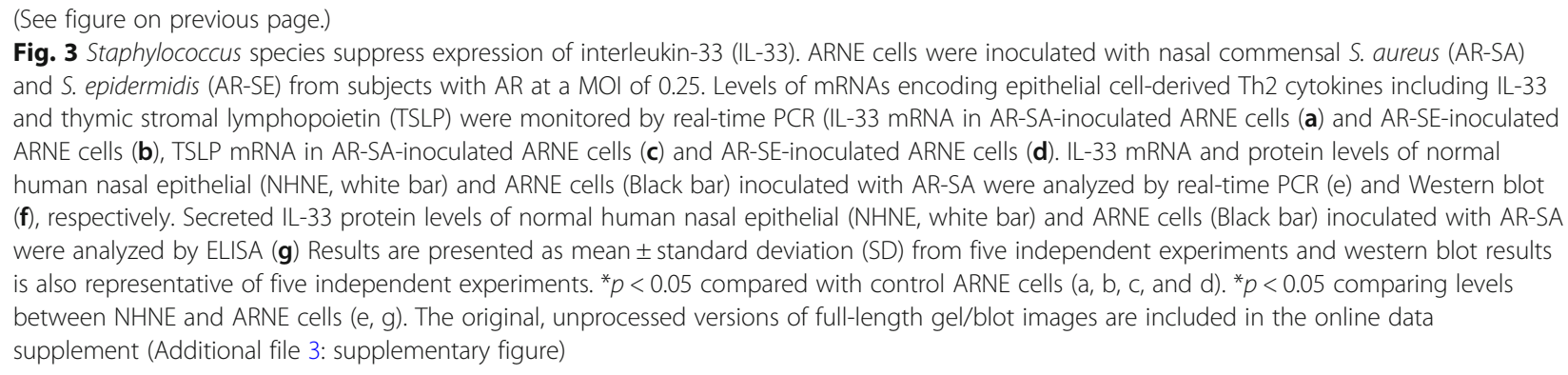

assessed the anti-allergic effect of $S$. aureus using an in vivo murine model of $A R$. BALB/C mice were sensitized and challenged with OVA (AR-OVA, $N=5$ ). BALB/C mice $(\mathrm{N}=5)$ were inoculated with human nasal microbiome $S$. aureus at 2 days (day 19, 20) following nasal microbiota depletion (day 15, 16, 17) using $30 \mu \mathrm{l}$ of an antibiotic cocktail (vancomycin, neomycin, ampicillin, and metronidazole) (Fig. 4a). Nasal symptoms such as sneezing and rubbing, total serum IgE, OVA-specific IgE, serum eosinophil count, and histologic findings of nasal mucosa were compared for WT and AR-OVA mice depending on human nasal AR-SA.

First, $S$. aureus mRNA levels were measured in in vivo nasal mucosa to confirm effective colonization of AR-SA in WT mice and AR-OVA mice after inoculation. RTPCR results revealed that femA mRNA levels and colony counts of $S$. aureus were significantly higher in WT and AR-OVA mice with $S$. aureus inoculation than in WT and AR-OVA groups without inoculation (Fig. 4b, c). The difference in allergic symptoms of in vivo AR mice was clearly observed depending on inoculation with $S$. aureus-inoculated AR-OVA mice. The frequencies of sneezing and nasal rubbing in the positive control group were $9.98 \pm 2.31$ (WT mice) and $17.01 \pm 1.43$ (AR-OVA), respectively. These scores were significantly reduced in AR-OVA mice with human nasal microbiome AR-SA inoculation (1.35 $\pm 0.61, p=0.018$, and $4.60 \pm 3.14, p=$ 0.010 , respectively) (Fig. $4 d$, e). Serum levels of total IgE (Fig. 4f) and OVA-specific IgE (Fig. 4g) were significantly lower in AR-OVA mice compared with the WT group. Eosinophils and secretory cells of nasal mucosal tissue were detected by Sirius red and PAS staining. Eosinophil infiltration in the submucosa of AR-OVA mice was significantly higher than that in $S$. aureus-inoculated AROVA mice (Fig. 4h). Fewer PAS-stained secretory cells were observed in nasal mucosal tissue of $S$. aureus-inoculated AR-OVA mice than in AR-OVA mice (Fig. 4i). Collectively, these data suggest that intranasal inoculation of human microbiome $S$. aureus improved allergic inflammation in an in vivo AR model. In addition, nasal microbiome isolated from allergic nasal mucus reduced Th2 inflammation of in vivo nasal mucosa.
Finally, mRNA expression of IL-4, IL-5, and IL-13 was measured to evaluate changes in Th2 cytokine-regulated inflammation of in vivo nasal mucosa after S. aureus inoculation. As expected, intranasal inoculation of AR-SA significantly reduced the mRNA levels of IL-4, IL-5, and IL-13 in in vivo nasal mucosa of AR-OVA mice compared to AR-OVA mice without AR-SA inoculation (Fig. 5a, b and c). Levels of IL-33 mRNA in mouse nasal mucosa were then determined by RT-PCR. Secreted IL33 protein levels in NAL fluid were also determined with ELISA in in vivo AR mice depending on human nasal $S$. aureus inoculation. RT-PCR results revealed a higher level of IL-33 mRNA in the nasal mucosa of AR-OVA mice that was significantly attenuated in the nasal mucosa of AR-OVA mice with AR-SA inoculation (Fig. 5d). In addition, OVA-induced secreted IL-33 protein level was significantly decreased in WT-OVA mice with inoculation of human nasal microbiome S. aureus (Fig. 5e). These findings demonstrate that the nasal mucosa of AR mice had significantly reduced Th2 cytokines in response to human nasal microbiome $S$. aureus. S. aureus-regulated anti-allergic immune responses might be involved in IL33 downregulation of the nasal mucosa in the in vivo AR model.

\section{Discussion}

Here, we showed that there was significantly more colonization by $S$. epidermidis and $S$. aureus in the nasal mucus of subjects with AR compared to healthy nasal mucus. In addition, inoculation of human nasal microbiome S. aureus to ARNE cells and an OVA-sensitized AR murine model reduced Th2 cytokine-regulated allergic inflammation by suppressing the epithelial cellderived cytokine IL-33. Our findings imply that intranasal administration of $S$. aureus is a potential strategy for improving allergic inflammation by regulating epithelial cell-derived cytokines. Inoculation of $S$. aureus isolated from nasal mucus of subjects with AR might be a new treatment for controlling allergic inflammation caused by inhaled allergens in the nasal epithelium.

Respiratory epithelium is the first target organ for environmental allergens, and recent work has highlighted 

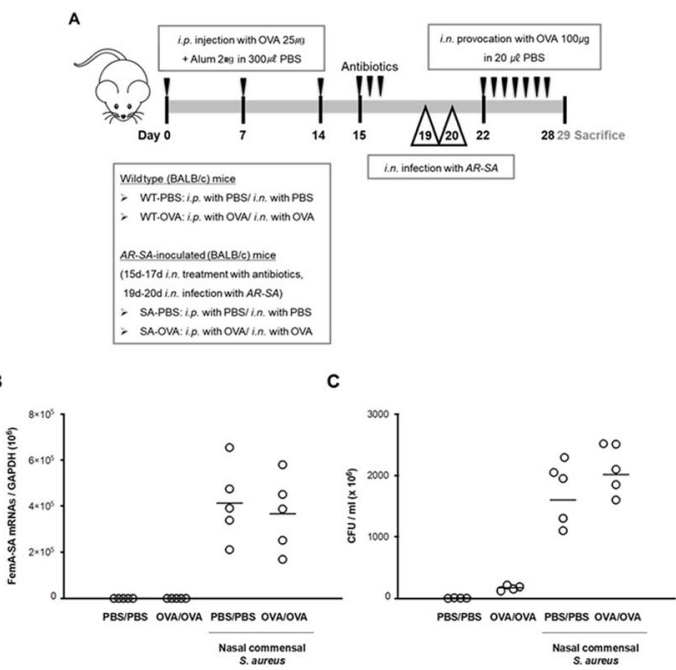

D
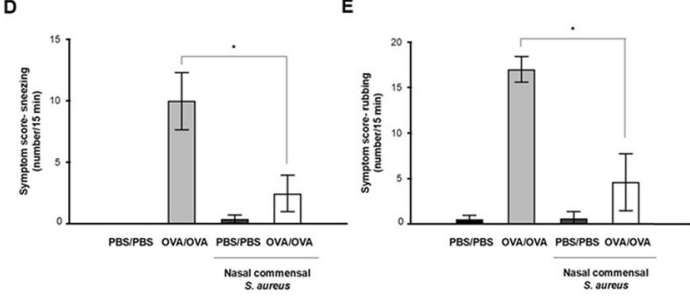

$\mathbf{F}$
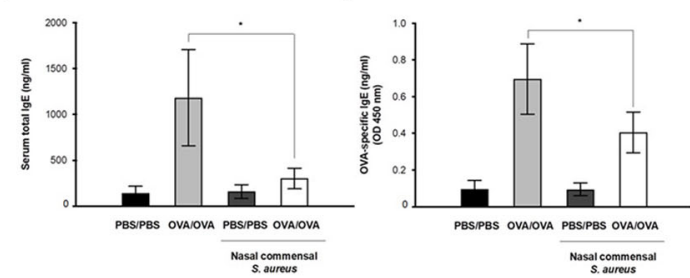

H
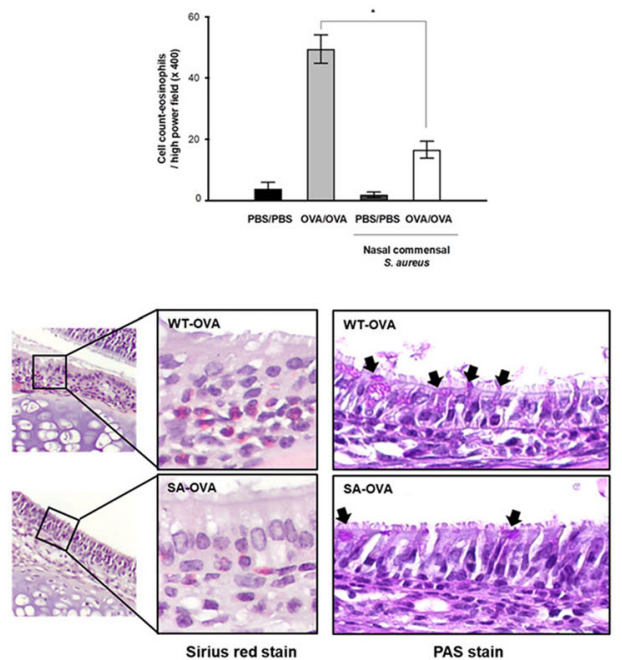

Fig. 4 Experimental protocol and comparison of nasal symptoms, serum immunoglobulin E (lgE) levels, and eosinophilic infiltration levels. a The experimental protocol for development of allergic asthmatic mouse using BALB/C. Mice were sensitized by intraperitoneal injection of ovalbumin mixed with aluminum hydroxide on days 0,7 , and 14. Daily OVA intranasal challenge was performed from days 22 to 28 (OVAVOVA). Human nasal S. aureus (AR-SA) $(3.2 \times$ $10^{6} \mathrm{CFU} / 30 \mu \mathrm{ul}$. PBS) was inoculated at indicated time points (day 19, 20). $\mathbf{b}$ mRNA levels of femA gene, specific for $S$. aureus and normalized to cellular GAPDH transcript levels, were monitored by real-time PCR. c Colony counts of nasal lavage fluid were performed. Frequencies of sneezing (d) and rubbing (e) events were assessed over a $15 \mathrm{~min}$ period after OVA provocation. Serum levels of total lgE (f) and OVAspecific IgE (g) were significantly lower in SA-OVA mice than in WTOVA mice $(N=5)$. Histologic findings in nasal mucosa of each group ( $\times 400$ magnification) with Sirius red staining for eosinophils (h) and periodic acid-Schiff (PAS) staining for secretory cells (i). Results are presented as mean \pm standard deviation $(\mathrm{SD})(\mathrm{N}=5)$ and histologic finding is representative of nose sections from five mice. ${ }^{*} p<0.05$

the critical role of the respiratory epithelium as a barrier that restricts host exposure of allergens [11, 21-23]. Like intestinal symbiotic commensal organisms, the microbiome that colonizes respiratory mucus is thought to have critical immune functions in the respiratory tract. There may be a functional connection between respiratory epithelial cells that contact inhaled allergens and the respiratory microbiome. There is increasing interest in compositional and predicted functional differences in respiratory mucus microbiomes of allergic diseases. The importance of the respiratory microbiome, especially with respect to Th2 cytokine-regulated immune responses, has been increasingly recognized [24-28]. Understanding compositional changes in the microbiome and identifying dominant microbial species in respiratory mucus might be essential for developing new therapeutic approaches for allergic respiratory diseases. The nasal mucosa is also a key player in immunologic defenses that protect the respiratory tract and is responsible for filtering inhaled allergens from direct exposure to pressurized airflow $[22,23]$.

Diverse evidence suggests that microbes reside in the nasal mucus secreted from epithelial cells. Our previous study revealed that the most dominant nasal microbiome, S. epidermidis, increased immunity against influenza viral lung infection through IFN- $\lambda$ amplification at the level of the nasal epithelium. The findings provide evidence that the nasal microbiome strengthens innate immune responses of the host to protect the respiratory tract from viral pathogens that it is exposed to [17]. We extended this paradigm to the nasal microbiome in the field of allergic diseases and noted marked changes in the microbial composition of nasal mucus in subjects with AR. The distribution of both $S$. epidermidis and $S$. aureus was significantly increased in AR nasal mucus to constitute over $60 \%$ of the identified nasal microbiome. 
A

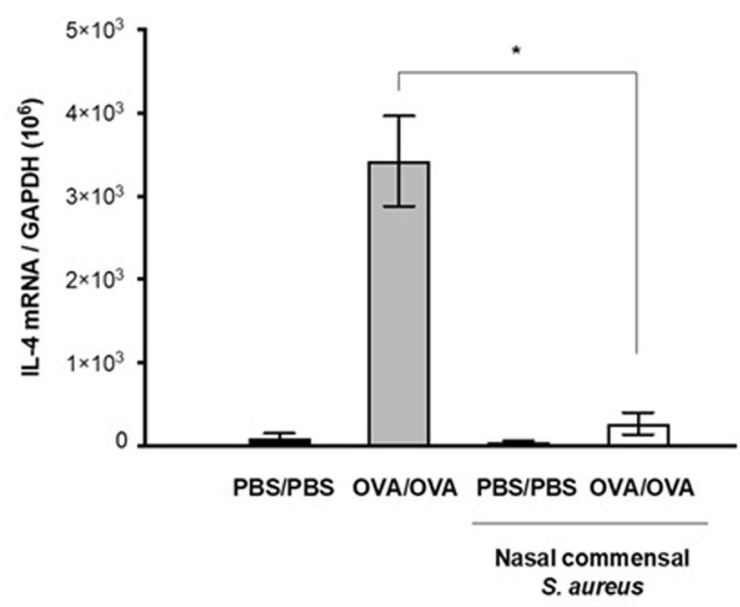

C

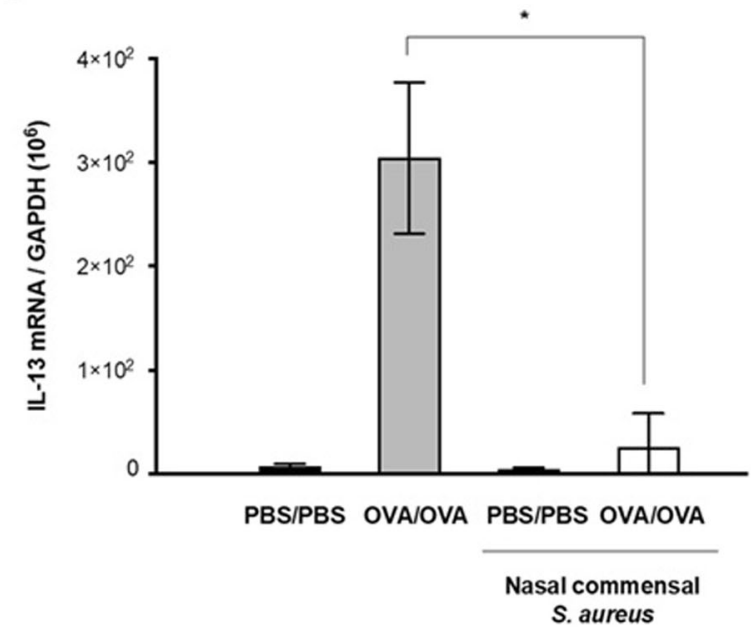

D

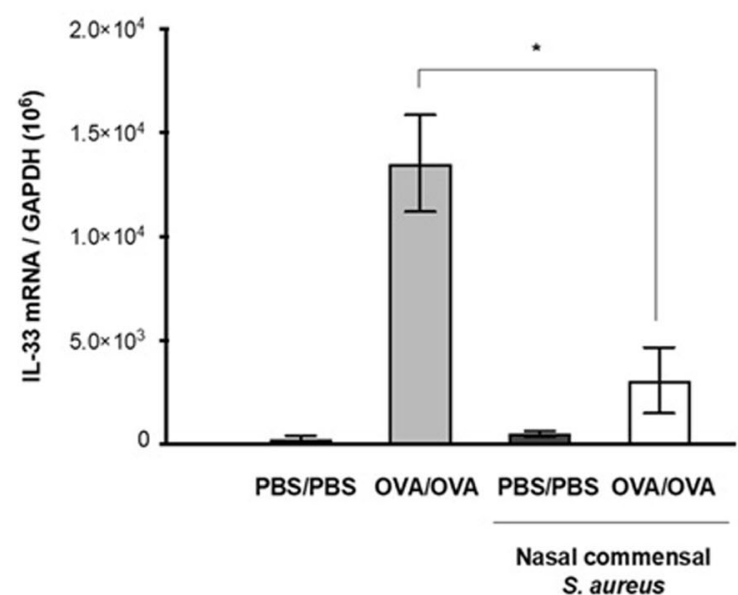

Fig. 5 (See legend on next page.)
B

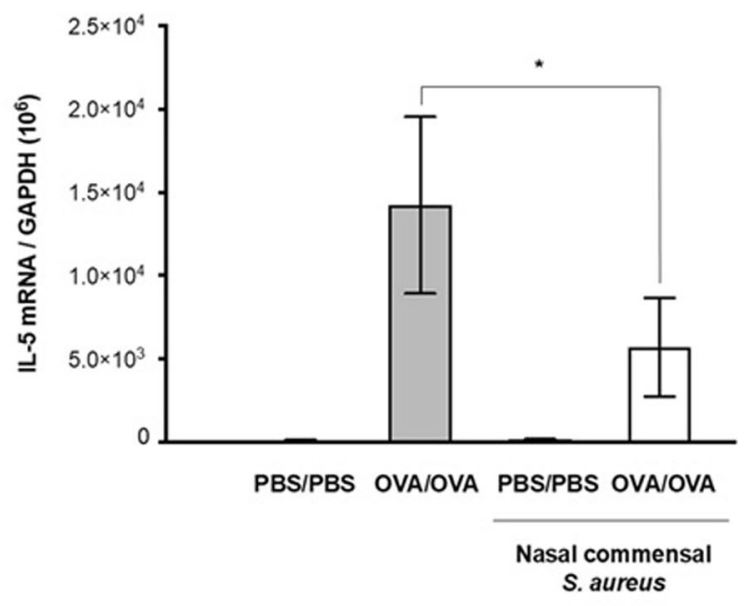

E

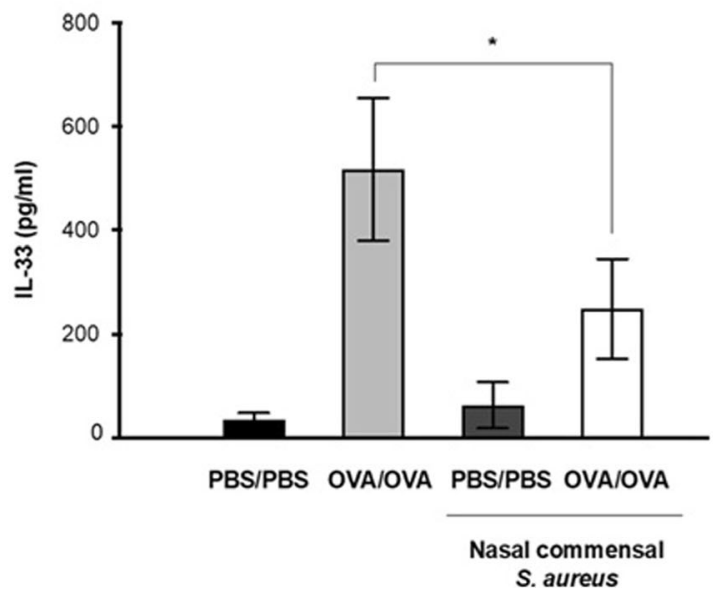


(See figure on previous page.)

Fig. 5 Comparison of cytokine mRNA levels in nasal mucosa. Wild type mice (PBS/PBS) and AR mice (OVA/OVA) were inoculated with human nasal S. aureus $\left(3.2 \times 10^{6} \mathrm{CFU} / 30 \mu \mathrm{lPBS}\right)$ at indicated time points (day 19, 20) and mRNA levels of interleukin (IL)-4 (a), IL-5 (b), IL-13 (c), and IL-33 (d) were measured by real-time PCR. e Secreted IL-33 protein levels secreted from nasal mucosa were measured by ELISA using nasal lavage fluid. Results are presented as mean \pm standard deviation (SD) $(N=5)$. ${ }^{*} p<0.05$

Among nasal microbiomes of AR nasal mucus, S. aureus colonization increased the most compared to normal mucus. Thus, we focused on the contributions of $S$. epidermidis and S. aureus to the pathogenic mechanism of AR and synergism of Staphylococcus species colonization with Th2-related allergic reaction in the nasal mucosa. Both S. epidermidis and S. aureus are well-known human pathogens that may cause serious opportunistic infections of the respiratory tract. However, Staphylococcus species are commonly detected in the normal microflora of the skin, intestine, and upper airway and many studies implicate the role of Staphylococcus species as symbiotic commensal organisms that are also involved in allergic diseases [29-31]. Patients with allergic eczema are more likely to have skin colonized with $S$. aureus than healthy control subjects, and disease severity is associated with $S$. aureus colonization. The dominant distribution of $S$. aureus in the upper respiratory tract of patients with asthma and aspirin sensitivity has been reported up to $87.5 \%$, and higher nasal $S$. aureus colonization is significantly related to asthma [29, 32]. In addition, patients with AR are more frequently colonized with nasal $S$. aureus or sensitized to $S$. aureus enterotoxins than healthy control subjects [30]. Therefore, we hypothesized that Staphylococcus species aggravate allergic immune responses in nasal epithelium, inoculation of Staphylococcus colonies dysregulates Th2 inflammation, and epithelial cell-derived Th2 cytokines of in vitro and in vivo AR models would be elevated. We investigated the definite function of Staphylococcus species in allergic nasal mucus by isolating colonies of $S$. epidermidis and S. aureus from human nasal mucus of subjects with AR.

Interestingly, the results differed from what was initially expected. Nasal commensal $S$. aureus isolated from the nasal mucus of subjects with AR reduced IL-33 expression in both ARNE cells and the OVA-driven AR mice model. Conversely, S. epidermidis isolated from the nasal mucus of subjects with AR did not induce significant changes in epithelial cell-derived Th2 cytokines. $S$. aureus induced reduction of IL-33 expression in nasal epithelium had a crucial impact on the downstream activities of epithelial cell-derived cytokines and suppressed allergic airway inflammation, eosinophil infiltration, and induction of Th2 cytokines such as IL-4, IL-5, and IL-13. This study demonstrates that colonization by $S$. epidermidis and $S$. aureus is highly increased in the nasal mucus of subjects with AR and particularly, inoculation of isolated nasal commensal $S$. aureus suppresses
Th2 cytokine-dependent allergic inflammation by regulating epithelial cell-derived cytokines, especially IL-33, in in vitro and in vivo AR models.

There are studies on the effect of the gut microbiome and bacterial administration on Th2 inflammation in AR. Gut microbiome organisms such as Lactobacilli and Bifidobacteria have recently been found to be effective in murine models of allergic airway inflammation. Animal studies have suggested the effects of gut commensal bacteria on allergen-induced airway responses, and administration of microbiome strains or bacterial components played a protective role in allergic airway inflammation [33, 34].

The nasal mucosa is a key player in immunologic responses to inhaled allergens, and we presumed that local microbiome distribution in the nasal mucus of subjects with AR might influence allergic inflammation. We wonder how nasal Staphylococcus species colonization is more abundant in AR nasal mucus and whether this plays a critical role in regulating Th2 immunity and allergic diseases. We surmise that allergic inflammation in nasal mucosa of subjects with AR is thought to provide a better environment for Staphylococcus species to colonize and inoculation of Staphylococcus species adapted to AR nasal mucus contributed to reduced Th2 inflammatory responses in allergic nasal mucosa. These findings propose that manipulation of the microbiome or inoculation of a dominant nasal microbiome might be a therapeutic strategy for AR.

Among various epithelial cell products, three epithelial cell-derived cytokines, IL-33, IL-25, and TSLP, have critical functions in the pathophysiology of allergic airway diseases. IL-33, predominantly expressed by tissue cells such as epithelial cells, fibroblasts, and endothelial cells, can be induced in allergic inflammatory conditions. By binding suppression of tumorigenicity 2 (ST2), IL-33 activates target cells to produce Th2 cytokines that regulate local and systemic allergic inflammation [6, 33]. Both IL-33 and ST2 are highly expressed in the nasal mucosa of subjects with AR, suggesting that the IL-33/ ST2 axis may be a key mechanistic link in the pathogenesis of allergic nasal diseases $[6,33]$. We suggest that reduction of IL-33 is a critical option for controlling inflammation in the nasal mucosa of patients with AR, and research to identify an effective modulator of IL-33 may improve the possibility of treating AR. We found that nasal commensal $S$. aureus isolated from subjects with AR preferentially modulated IL-33 expression in 
both ARNE cells and the nasal mucosa of an OVAdriven AR mice model. The current data indicate that isolation of $S$. aureus from the nasal mucus of subjects with $\mathrm{AR}$ and culturing it for intranasal inoculation is a promising therapeutic option for restricting IL-33/ST signaling pathways in AR nasal mucosa. Although $S$. aureus may cause respiratory infections, isolation of $S$. aureus from the adapted environment of AR nasal mucus reduced Th2 inflammation by suppressing IL-33 expression in allergic nasal epithelium.

Our study increases understanding of whether the nasal microbiome of subjects with AR reduces Th2 inflammation to protect AR nasal epithelium from IL-33-mediated inflammation. We showed that abundant $S$. aureus colonization enhances resistance to allergic inflammation in human nasal epithelium by reducing IL-33 expression in nasal mucosa. Thus, intranasal delivery of human nasal commensal $S$. aureus represents a potential therapeutic approach for preventing and treating allergic inflammation via reduction of epithelial cell-derived Th2 cytokines.

\section{Supplementary information}

Supplementary information accompanies this paper at https://doi.org/10. 1186/s12866-020-01974-6.

Additional file 1. A video file of the nasal swab for sample collection. Additional file 2. Supplementary material and method.

Additional file 3. Supplementary figure.

\section{Abbreviations}

AR: Allergic rhinitis; AR-SA: Staphylococcus aureus; AR-SE: Staphylococcus epidermidis; NHNE: Normal human nasal epithelium; ARNE: Allergic rhinitis nasal epithelium; TSLP: Thymic stromal lymphopoietin; IL-33: Interleukin-33; Th2: $T$ helper 2; Tregs: Regulatory $T$ cells

\section{Acknowledgements}

Not applicable.

\section{Authors' contributions}

Study conception and design: YJJ and HJK. Data acquisition: YJJ, CHG, JW, AJ, and HJK. Data analysis and interpretation: YJJ and HJK. Drafting the article or critically revising it for important intellectual content: YJJ and HJK. Final approval of the version to be submitted: HJK. All authors have contributed and approved the final manuscript.

\section{Funding}

This work was supported by the Basic Science Research Program through the National Research Foundation of Korea (NRF) funded by the Ministry of Education (2016R1D1A1B01014116 to HJK) and by the Collaborative Genome Program for Fostering New Post-Genome Industry of the National Research Foundation (NRF) funded by the Ministry of Science and ICT (MSIT) (2019M3C9A6091942, 2019M3C9A6091945 to HJK). This research was also supported by a grant of the Basic Science Research Program through the National Research Foundation of Korea (NRF) funded by the Ministry of Education (2019R1C1C1002064 to YJJ) and by biomedical research institute fund (GNUHBRIF-2020-0007 to YJJ) from the Gyeongsang National University Hospital. The funders had no role in study design, data collection and analysis, decision to publish, or preparation of the manuscript.

\section{Availability of data and materials}

The data that support the findings of the current study are available from the corresponding author upon reasonable request.

\section{Ethics approval and consent to participate}

This study was approved and monitored by the Institutional Review Board (IRB) of Seoul National University College of Medicine (No. 1709-049-883) and Gyeongsang National University Hospital (No. 2019-05-004), and by the Institutional Animal Care and Use Committees of Seoul National University Hospital (No. 2016-1470). All research methods were carried out in accordance with approved guidelines. Participation was voluntary, and written informed consent was obtained from all subjects.

Consent for publication

Not applicable.

\section{Competing interests}

The authors declare that the research was conducted in the absence of any commercial or financial relationships that could be construed as potential conflicts of interest.

\section{Author details}

${ }^{1}$ Department of Otorhinolaryngology, Gyeongsang National University Hospital, Jinju, Republic of Korea. ${ }^{2}$ Department of Otorhinolaryngology Head and Neck Surgery, Seoul National University College of Medicine, 103, Daehak-ro, Jongno-gu, Seoul 03080, Republic of Korea. ${ }^{3}$ Department of Otorhinolaryngology, Seoul National University Hospital, Seoul, Republic of Korea.

Received: 26 April 2020 Accepted: 10 September 2020

Published online: 07 October 2020

\section{References}

1. Won J, Gil CH, Jo A, Kim HJ. Inhaled delivery of interferon-lambda restricts epithelial-derived Th2 inflammation in allergic asthma. Cytokine. 2019;119: 32-6. https://doi.org/10.1016/j.cyto.2019.02.010.

2. Hammad H, Lambrecht BN. Barrier epithelial cells and the control of type 2 immunity. Immunity. 2015;43(1):29-40. https://doi.org/10.1016/j.immuni. 2015.07.007.

3. Saenz SA, Taylor BC, Artis D. Welcome to the neighborhood: epithelial cellderived cytokines license innate and adaptive immune responses at mucosal sites. Immunol Rev. 2008;226:172-90. https://doi.org/10.1111/j.1600065X.2008.00713.X

4. Jeon YJ, Lim JH, An S, Jo A, Han DH, Won TB, et al. Type III interferons are critical host factors that determine susceptibility to influenza a viral infection in allergic nasal mucosa. Clin Exp Allergy. 2018;48(3):253-65. https://doi.org/ 10.1111/cea.13082.

5. Takatori H, Makita S, Ito T, Matsuki A, Nakajima H. Regulatory mechanisms of IL-33-ST2-mediated allergic inflammation. Front Immunol. 2018;9:2004. https://doi.org/10.3389/fimmu.2018.02004

6. Drake LY, Kita H. IL-33: biological properties, functions, and roles in airway disease. Immunol Rev. 2017;278(1):173-84. https://doi.org/10.1111/imr.12552.

7. Ding T, Schloss PD. Dynamics and associations of microbial community types across the human body. Nature. 2014;509(7500):357-60. https://doi. org/10.1038/nature13178.

8. Erturk-Hasdemir D, Kasper DL. Resident commensals shaping immunity. Curr Opin Immunol. 2013;25(4):450-5. https://doi.org/10.1016/j.coi.2013.06.001.

9. Brestoff $J R$, Artis D. Commensal bacteria at the interface of host metabolism and the immune system. Nat Immunol. 2013;14(7):676-84. https://doi.org/ 10.1038/ni.2640

10. Hinks TS. Mucosal-associated invariant T cells in autoimmunity, immunemediated diseases and airways disease. Immunology. 2016;148(1):1-12. https://doi.org/10.1111/imm.12582.

11. Kim HJ, Kim CH, Ryu JH, Kim MJ, Park CY, Lee JM, et al. Reactive oxygen species induce antiviral innate immune response through IFN-lambda regulation in human nasal epithelial cells. Am J Respir Cell Mol Biol. 2013; 49(5):855-65. https://doi.org/10.1165/rcmb.2013-00030C.

12. Segal LN, Rom WN, Weiden MD. Lung microbiome for clinicians. New discoveries about bugs in healthy and diseased lungs. Ann Am Thorac Soc. 2014;11(1):108-16. https://doi.org/10.1513/AnnalsATS.201310-339FR.

13. Hilty M, Burke C, Pedro H, Cardenas P, Bush A, Bossley C, et al. Disordered microbial communities in asthmatic airways. PLoS One. 2010;5(1):e8578. https://doi.org/10.1371/journal.pone.0008578. 
14. Huffnagle GB, Dickson RP, Lukacs NW. The respiratory tract microbiome and lung inflammation: a two-way street. Mucosal Immunol. 2017;10(2):299-306. https://doi.org/10.1038/mi.2016.108.

15. Wesemann DR, Nagler CR. The microbiome, timing, and barrier function in the context of allergic disease. Immunity. 2016;44(4):728-38. https://doi.org/ 10.1016/.j.immuni.2016.02.002.

16. Suzaki H, Watanabe S, Pawankar R. Rhinosinusitis and asthma-microbiome and new perspectives. Curr Opin Allergy Clin Immunol. 2013;13(1):45-9. https://doi.org/10.1097/ACl.0b013e32835b34f6.

17. Kim HJ, Jo A, Jeon YJ, An S, Lee KM, Yoon SS, et al. Nasal commensal Staphylococcus epidermidis enhances interferon-lambda-dependent immunity against influenza virus. Microbiome. 2019;7(1):80. https://doi.org/ 10.1186/s40168-019-0691-9.

18. Lehtinen MJ, Hibberd AA, Mannikko S, Yeung N, Kauko T, Forssten S, et al. Nasal microbiota clusters associate with inflammatory response, viral load, and symptom severity in experimental rhinovirus challenge. Sci Rep. 2018; 8(1):11411. https://doi.org/10.1038/s41598-018-29793-w.

19. Park DY, Kim S, Kim CH, Yoon JH, Kim HJ. Alternative method for primary nasal epithelial cell culture using intranasal brushing and feasibility for the study of epithelial functions in allergic rhinitis. Allergy Asthma Immunol Res. 2016;8(1):69-78. https://doi.org/10.4168/aair.2016.8.1.69

20. Quan SH, Zhang YL, Han DH, Iwakura Y, Rhee CS. Contribution of interleukin $17 \mathrm{~A}$ to the development and regulation of allergic inflammation in a murine allergic rhinitis model. Ann Allergy Asthma Immunol. 2012;108(5): 342-50. https://doi.org/10.1016/j.anai.2012.02.014.

21. Kim S, Kim MJ, Kim CH, Kang JW, Shin HK, Kim DY, et al. The superiority of IFN-lambda as a therapeutic candidate to control acute influenza viral lung infection. Am J Respir Cell Mol Biol. 2017;56(2):202-12. https://doi.org/10. 1165/rcmb.2016-0174OC.

22. Sahin-Yilmaz A, Naclerio RM. Anatomy and physiology of the upper aimway. Proc Am Thorac Soc. 2011;8(1):31-9. https:/doi.org/10.1513/pats.201007-050RN.

23. Giacchi RJ, Lebowitz RA, Jacobs JB. Middle turbinate resection: issues and controversies. Am J Rhinol. 2000;14(3):193-7. https://doi.org/10.2500/ 105065800782102726.

24. Planet PJ, Parker D, Cohen TS, Smith H, Leon JD, Ryan C, et al. Lambda Interferon Restructures the Nasal Microbiome and Increases Susceptibility to Staphylococcus aureus Superinfection. mBio. 2016;7(1):e01939-15. https://doi. org/10.1128/mBio.01939-15

25. Tarabichi Y, Li K, Hu S, Nguyen C, Wang X, Elashoff D, et al. The administration of intranasal live attenuated influenza vaccine induces changes in the nasal microbiota and nasal epithelium gene expression profiles. Microbiome. 2015;3:74. https://doi.org/10.1186/s40168-015-0133-2.

26. Bassis CM, Tang AL, Young VB, Pynnonen MA. The nasal cavity microbiota of healthy adults. Microbiome. 2014;2:27. https://doi.org/10.1186/2049-2618-2-27.

27. Ramakrishnan VR, Feazel LM, Gitomer SA, Ir D, Robertson CE, Frank DN. The microbiome of the middle meatus in healthy adults. PLoS One. 2013;8(12): e85507. https://doi.org/10.1371/journal.pone.0085507.

28. Beck JM, Young VB, Huffnagle GB. The microbiome of the lung. Transl Res. 2012;160(4):258-66. https://doi.org/10.1016/j.trsl.2012.02.005.

29. Tsilochristou O, du Toit G, Sayre PH, Roberts G, Lawson K, Sever ML, et al. Association of Staphylococcus aureus colonization with food allergy occurs independently of eczema severity. J Allergy Clin Immunol. 2019;144(2):494503. https://doi.org/10.1016/j.jaci.2019.04.025.

30. Krismer B, Weidenmaier C, Zipperer A, Peschel A. The commensal lifestyle of Staphylococcus aureus and its interactions with the nasal microbiota. Nat Rev Microbiol. 2017;15(11):675-87. https://doi.org/10.1038/nrmicro.2017.104.

31. Riechelmann H, Essig A, Deutschle T, Rau A, Rothermel B, Weschta M. Nasal carriage of Staphylococcus aureus in house dust mite allergic patients and healthy controls. Allergy. 2005;60(11):1418-23. https://doi.org/10.1111/j.13989995.2005.00902.x.

32. Kim YC, Won HK, Lee JW, Sohn KH, Kim MH, Kim TB, et al. Staphylococcus aureus nasal colonization and asthma in adults: systematic review and meta-analysis. J Allergy Clin Immunol Pract. 2019;7(2):606-15 e9. https://doi. org/10.1016/j.jaip.2018.08.020

33. Ding W, Zou GL, Zhang W, Lai XN, Chen HW, Xiong LX. Interleukin-33: Its Emerging Role in Allergic Diseases. Molecules. 2018;23(7). https://doi.org/10. 3390/molecules23071665.

\section{Publisher's Note}

Springer Nature remains neutral with regard to jurisdictional claims in published maps and institutional affiliations.

Ready to submit your research? Choose BMC and benefit from:

- fast, convenient online submission

- thorough peer review by experienced researchers in your field

- rapid publication on acceptance

- support for research data, including large and complex data types

- gold Open Access which fosters wider collaboration and increased citations

- maximum visibility for your research: over $100 \mathrm{M}$ website views per year

At BMC, research is always in progress.

Learn more biomedcentral.com/submissions 